\title{
The eccentric short-period orbit of the supergiant fast X-ray transient HD 74194 (=LM Vel)^
}

\author{
R. Gamen ${ }^{1}$, R. H. Barbá ${ }^{2}$, N. R. Walborn ${ }^{3}$, N. I. Morrell ${ }^{4}$, J. I. Arias², J. Maíz Apellániz ${ }^{5}$, A. Sota ${ }^{6}$, and E. J. Alfaro ${ }^{6}$ \\ ${ }^{1}$ Instituto de Astrofísica de La Plata, CCT La Plata-CONICET, Facultad de Ciencias Astronómicas y Geofísicas, \\ Universidad Nacional de La Plata, Paseo del Bosque S/N, (1900) La Plata, Argentina \\ e-mail: rgamen@fcaglp.unlp.edu.ar \\ 2 Departamento de Física, Universidad de La Serena, Av. Juan Cisternas 1200 Norte, La Serena, Chile \\ 3 Space Telescope Science Institute ${ }^{\star \star}, 3700$ San Martin Drive, Baltimore, MD 21218, USA \\ 4 Las Campanas Observatory, Carnegie Observatories, Casilla 601, La Serena, Chile \\ 5 Centro de Astrobiología, CSIC-INTA, campus ESAC, apartado postal 78, 28691 Villanueva de la Cañada, Madrid, Spain \\ ${ }^{6}$ Instituto de Astrofísica de Andalucía-CSIC, Glorieta de la Astronomía s/n, 18008 Granada, Spain
}

Received 7 August 2015 / Accepted 12 October 2015

\section{ABSTRACT}

\begin{abstract}
Aims. We present the first orbital solution for the O-type supergiant star HD 74194, which is the optical counterpart of the supergiant fast X-ray transient IGR J08408-4503.

Methods. We measured the radial velocities in the optical spectrum of HD 74194, and we determined the orbital solution for the first time. We also analysed the complex $\mathrm{H} \alpha$ profile.

Results. HD 74194 is a binary system composed of an O-type supergiant and a compact object in a short-period $(P=9.5436 \pm$ $0.0002 \mathrm{~d})$ and high-eccentricity ( $e=0.63 \pm 0.03$ ) orbit. The equivalent width of the H $\alpha$ line is not modulated entirely with the orbital period, but seems to vary in a superorbital period $(P=285 \pm 10 \mathrm{~d})$ nearly 30 times longer than the orbital one.
\end{abstract}

Key words. binaries: spectroscopic - stars: early-type - stars: individual: HD 74194

\section{Introduction}

HD 74194 (=LM Vel) is a supergiant O-type star (O8.5 Ib-II(f)p; Sota et al. 2014) ${ }^{1}$. It was first suspected of binarity by Gies (1987), based on the two radial-velocity (RV) measurements published by Feast et al. $\left(1955,-4.3 \mathrm{~km} \mathrm{~s}^{-1}\right)$ and Conti et al. $\left(1977,22.4 \mathrm{~km} \mathrm{~s}^{-1}\right)$. No further analysis was found in the literature.

Because the binary nature of HD 74194 was uncertain, we included it in the sample of a RV monitoring programme. This project, named the OWN Survey, is carrying out systematic spectroscopic observations of all the O- and WN-type stars known in our Galaxy (Barbá et al. 2010), for which there was no or weak indication of binarity or multiplicity. The major goals are to search for RV variations that are indicative of orbital motion and to derive orbital solutions for as many of the variables as possible. The project also aims at building a high-quality spectral library of O-type stars to be used as a first epoch of RVs for future searches and also as spectral variability studies. The current sample of the OWN Survey includes the southern O-type stars from version 1.0 of the Galactic O-Star Catalog (Maíz Apellániz et al. 2004) and some Wolf-Rayet stars of the nitrogen sequence (WN) from the 7th Catalog of Galactic WR Stars (van der Hucht 2001). The OWN Survey started in 2005, and it has collected more than 6000 high-resolution spectrograms to date.

* Table 3 is available in electronic form at http://www . aanda.org $\star \star$ Operated by AURA, Inc., under NASA contract NAS5-2655.

1 HD 74194 was first identified as a supergiant by Walborn (1973), who classified it as $\mathrm{O} 8.5 \mathrm{Ib}(\mathrm{f})$.
Gotz et al. (2006) report the discovery of an INTEGRAL source, IGR J08408-4503, which they associated with HD 74194, although they could not discard the possibility of it being a very long and soft gamma-ray burst (GRB) at high redshift. Almost immediately, Mereghetti et al. (2006) found a previous, unreported outburst in the INTEGRAL archive, making the GRB hypothesis highly unlikely. They identified the source as a supergiant fast X-ray transient (SFXT), just because of the presence of the supergiant HD 74194. A similar conclusion was reached by Masetti et al. (2006). They analysed ESO optical archival spectra, and the presence of $\mathrm{H} \alpha$ in emission justified the optical-X-ray connection. At that time, the OWN Survey was beginning, and a few optical spectra were available. We analysed them and detected variability in both the $\mathrm{H} \alpha$ profiles and the RVs, which pointed to a binary nature. We therefore reported observational evidence to interpret HD 74194 as a SFXT object (Barbá et al. 2006).

We continued observing the optical spectrum of HD 74194 within the OWN Survey programme until this year, and we found a definitive orbital solution. Although several studies were published following the initial rapid sequence of telegrams (between 18 and 23 May 2006) analysing the hard X-ray source as a SFXT, this paper provides the first direct proof that HD 74194 is a binary system and thus that the identification is correct.

SFXTs are supposed to be binary systems comprised of a blue supergiant star and a compact (usually neutron-star) object displaying extreme transient flaring activity in the X-ray domain (see the review of Sidoli 2013 for details). This more extreme X-ray variability is the criterion proposed to distinguish SFXTs from the "classical" supergiant X-ray binaries, which 
Table 1. Details of the spectroscopic data for HD 74194.

\begin{tabular}{llccc}
\hline \hline Instr. config. & Observatory & $R$ & $\begin{array}{c}\text { Sp. range } \\
{[\mathrm{nm}]}\end{array}$ & $N^{a}$ \\
\hline Echelle, 2.5-m & LCO & 45000 & $345-985$ & 23 \\
REOSC, 2.15-m & CASLEO & 15000 & $360-610$ & 14 \\
FEROS, 2.2-m & La Silla & 46000 & $357-921$ & 19 \\
\hline
\end{tabular}

Notes. ${ }^{(a)} N$ is the number of spectra.

share other macroscopic properties such as the range of orbital periods (Bozzo et al. 2015). The mystery of SFXTs is the origin of this difference. To date, there are only twelve confirmed SFXTs listed in Romano et al. (2014) and Romano (2015). IGR J08408-4503 = HD 74194 is one of the three that display the broadest X-ray dynamic ranges $\left(\geq 10^{4}-10^{5}\right)$ and that are indicated as an "SFXT prototype" (Bozzo et al. 2015).

In this work, we present the optical spectroscopic observations and the RV orbit of HD 74194. We also study the coincidence of the reported flares with the periastron passages, which strongly suggests a link between the two events. In addition, we study the variability of the $\mathrm{H} \alpha$ profile, searching for a superorbital period.

\section{Observations}

This work is based on spectroscopic observations with highresolution spectrographs at Complejo Astronómico El Leoncito (CASLEO) in Argentina, and at the Las Campanas (LCO) and La Silla (ESO) Observatories in Chile. A summary of the spectral characteristics is provided in Table 1.

We obtained a calibration lamp exposure immediately before or after each target integration in the same telescope position when the spectrograph is attached to the telescope (i.e. the ones at CASLEO and LCO). The spectra were processed and calibrated using standard routines in $\mathrm{IRAF}^{2}$, except FEROS data, for which we applied the standard reduction pipeline provided by ESO (which uses comparison lamps observed before and after each night).

\section{The radial-velocity orbit}

We measured the central wavelengths of the He II $\lambda \lambda 4542,4686$, and 5411 absorption lines in the 56 spectra of HD 74194 and determined their RVs by means of the SPLOT task in IRAF. We chose these three lines because they present very similar RVs in a given spectrum. Other lines, such as He I $\lambda \lambda 4471$ and 5875, present profile variations that prevent appropriate RV measurements.

The RVs are clearly variable (as indicated by the first observations, Barbá et al. 2006). We therefore searched for periodicities in the whole RV dataset, and using the code published by Marraco \& Muzzio (1980), we obtained a most probable period $P=9.543$ days. A very similar result of 9.542 days was given by the Lomb-Scargle algorithm (Scargle 1982) provided online as a NASA Exoplanet Archive service (Akeson et al. 2013).

\footnotetext{
2 IRAF is distributed by the National Optical Astronomy Observatories, which are operated by the Association of Universities for Research in Astronomy, Inc., under cooperative agreement with the National Science Foundation.
}

Table 2. Orbital elements of HD 74194 determined by two independent methods.

\begin{tabular}{lcc}
\hline \hline Parameter & GBART & FOTEL \\
\hline$P[\mathrm{~d}]$ & $9.5436 \pm 0.0002$ & $9.5436 \pm 0.0002$ \\
$e$ & $0.63 \pm 0.03$ & $0.63 \pm 0.05$ \\
$T_{\text {periastron }}[\mathrm{HJD}]^{a}$ & $634.95 \pm 0.04$ & $634.95 \pm 0.05$ \\
$T_{\mathrm{VRmax}}\left[\mathrm{km} \mathrm{s}^{-1}\right]$ & $635.27 \pm 0.04$ & $635.27 \pm 0.05$ \\
$\omega\left[{ }^{\circ}\right]$ & $302 \pm 4$ & $302 \pm 4$ \\
$V_{0}\left[\mathrm{~km} \mathrm{~s}^{-1}\right]$ & $15.3 \pm 0.4$ & $15.3 \pm 0.5$ \\
$K\left[\mathrm{~km} \mathrm{~s}^{-1}\right]$ & $21 \pm 1$ & $20.8 \pm 1.4$ \\
$a \sin i\left[R_{\odot}\right]$ & $3.03 \pm 0.24$ & $3.1 \pm 0.3$ \\
$F(M)\left[M_{\odot}\right]$ & $0.004 \pm 0.001$ & $0.0042 \pm 0.0015$ \\
$\mathrm{rms}\left[\mathrm{km} \mathrm{s}^{-1}\right]$ & 2.3 & 3.4 \\
\hline
\end{tabular}

Notes. ${ }^{(a)}$ Heliocentric Julian day-2 454000.

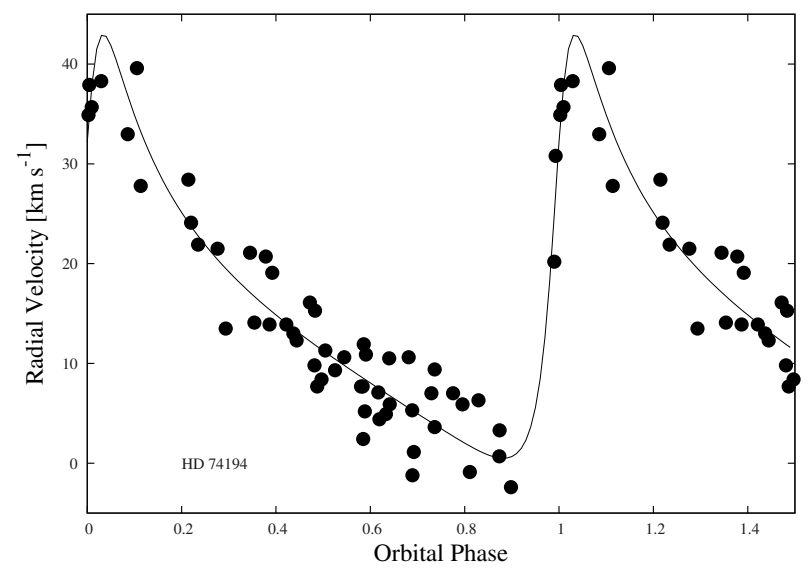

Fig. 1. Radial-velocity orbit of HD 74194 derived from He II lines.

We determined the RV orbital solution by means of the GBART code $^{3}$. This code converges to a highly eccentric orbit, $e=0.63 \pm 0.04$ and $P=9.5436 \pm 0.0002 \mathrm{~d}$. We checked the GBART orbital solution by means of the FOTEL code developed by Hadrava (2004), obtaining the same solution. The orbital elements determined by both methods are shown in Table 2 . Thus, the new ephemeris of the system, in heliocentric Julian days, is

$T_{\text {periastron }}=2454654.04+9.5436 \times E$.

In addition, we took advantage of the FOTEL code to explore the possibility that this high-eccentricity system might display apsidal motion. We tried solutions with the rate of apsidal advance $(\dot{\omega})$ as a free parameter, and also split the RVs into two datasets covering the years 2006-2009 and 2010-2015. Neither attempt gave definitive conclusions. This kind of analysis should be done with future observations with our solution as the initial epoch.

\section{Variability of the $\mathrm{H} \alpha$ line}

We collected 42 spectra that cover the $\mathrm{H} \alpha$ line. The line is variable on a timescale of days in both intensity and the RV of the absorption and emission components. In most of the spectra, the $\mathrm{H} \alpha$ line exhibits $\mathrm{P}$ Cygni-like profiles, with an additional extended emission in the blue wing (described as a blue hump or

\footnotetext{
3 GBART is an improved version of the original program for determining the orbital elements for spectroscopic binaries (Bertiau \& Grobben 1969) developed by F. Bareilles and available at http://www.iar. unlp. edu. ar/ fede/pub/gbart
} 
R. Gamen et al.: The eccentric short-period orbit of the supergiant fast X-ray transient HD 74194 (=LM Vel)

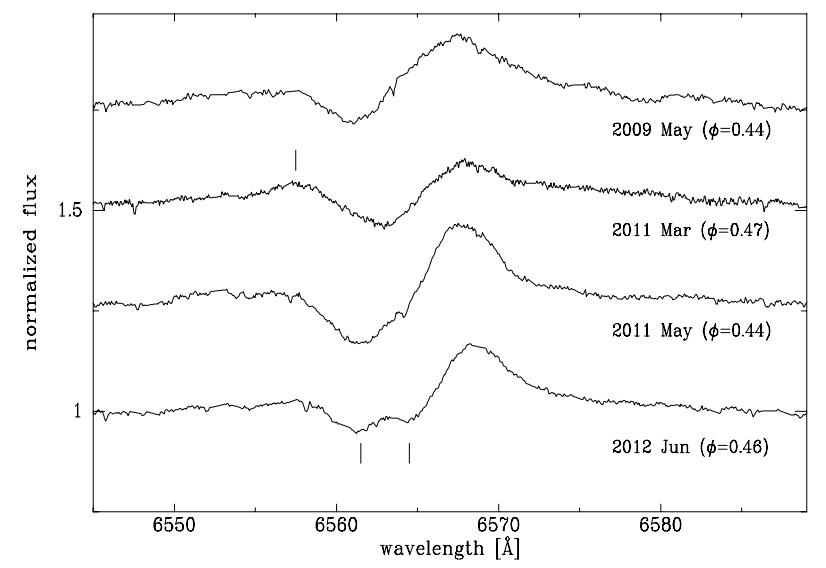

Fig. 2. Variability of $\mathrm{H} \alpha$ in the spectrum of HD 74194 at similar orbital phases.

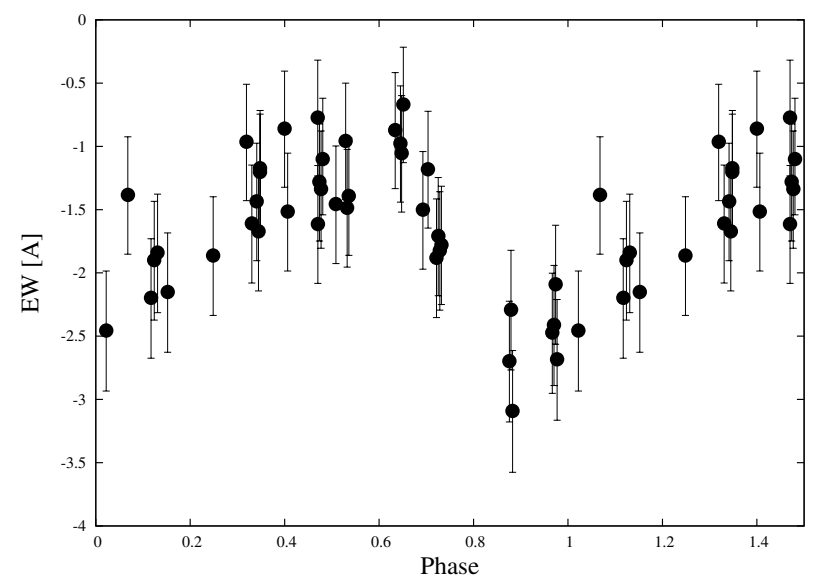

Fig. 3. Variation in the EW of $\mathrm{H} \alpha$, phased with a period of 285.3 days and the time of periastron passage of the RV orbit. Bars represent the errors in the EWs.

Beal's Type III P Cygni profile). In a few spectra, the absorption is located near the centre of the emission profile, producing an apparent double emission with two peaks with similar intensities.

We measured the RVs of the absorption and emission components in each spectrum, whenever possible. The RVs of the emission peaks folded with the orbital ephemeris of Eq. (1) roughly follow the same trend as the RVs of the He II absorption lines, although they show a larger dispersion with a semiamplitude of $K=50 \pm 10 \mathrm{~km} \mathrm{~s}^{-1}$. This behaviour suggests that the $\mathrm{H} \alpha$ emission is associated with the O-type star. On the other hand, the RVs of the absorption components do not follow the orbital period, and no other reliable periodicity was found (via the MARMUZ program or the NASA Exoplanet Archive Periodogram Service). The material producing this absorption is not bound to the O-type star or to the secondary star.

We investigated whether the morphological variability of the complex $\mathrm{H} \alpha$ line is related to the orbital period by comparing spectra taken at the same orbital phases. For example, in Fig. 2, we compare four $\mathrm{H} \alpha$ spectra taken at different epochs but similar orbital phases. The spectrum obtained in 2012 June exhibits a P-Cygni profile with a double absorption component, whereas in the other spectra, the P-Cyg profiles present a single absorption component. Also, the extended emission in the blue wing (blue hump) of the 2011 March spectrum is notable. Thus, the profile variability in $\mathrm{H} \alpha$ is not entirely modulated with the orbital period.

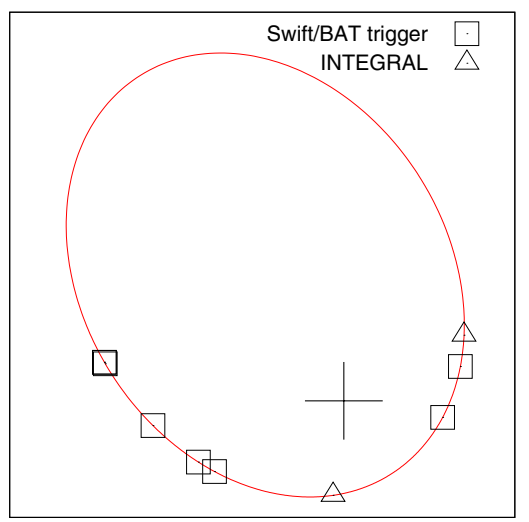

Fig. 4. Positions, relative to the barycentre, of the O-type supergiant during the outbursts of HD 74194 found in the literature.

We also analysed the variability in the equivalent widths (EW) of the complex $\mathrm{H} \alpha$ line. Such measurements are problematic because it is difficult to define the continuum in the echelle orders. We used the continuum of the next bluer order, which has no conspicuous spectral lines, and divided the order including $\mathrm{H} \alpha$ by it (pixel to pixel). The spectra were also corrected by their respective RVs of the $\mathrm{H} \alpha$ emission. Then, we determined the EW in the resulting spectra, using the SPLOT task in IRAF in script mode to fix the wavelength limits between $6535 \AA$ and $6580 \AA$ (thus including absorption and emission components). We searched for periodicities in the obtained EWs with the NASA service. We obtained a most probable period of $P=$ $285.3 \mathrm{~d}$, which is nearly 30 times the RV period. The period uncertainty can be estimated as the full width at half maximum of the periodogram peak (relative to power values); in this case, the result is about ten days.

\section{Discussion and conclusions}

We present for the first time the orbital solution of HD 74194, based on the RVs of optical absorption lines of He II measured in 56 spectra secured between 2006 and 2015. The resulting orbit displays a high eccentricity $(e=0.63 \pm 0.03)$ and a rather short periodicity $(P=9.5436 \mathrm{~d})$. Such a combination of parameters is well outside the known distribution, in which there is no binary system with $P<10$ days and $e>0.43$, the most extreme previously known system being HD $37737(P=7.84 \mathrm{~d}$ and $e=0.43$, McSwain et al. 2007). Such close and highly eccentric binary orbits are thought to be possible in post-SN systems (Kalogera 1996).

The new orbital ephemeris for HD 74194 supports analysis of the reported high-energy outbursts according to their phases. We summarised these events in the Table 3, where their respective orbital phases (according to the ephemeris of Eq. (1)) are also shown. It is remarkable that they occur in a restricted range of orbital phases, namely $\phi=0.84-0.07$, which is very near to the times of the periastron passage $(\phi=0.00)$. The apparent correlation between flares and periastron passages (see Fig. 4) seems to agree with the scenario of a compact star accreting matter from the clumpy wind of the supergiant. Karino (2010) proposes different scenarios for this phenomenon, relating $P$ and $e$. HD 74194 falls into the SFXT regime, but very near to the unstable orbit region, where stars could evolve towards coalescence.

We can discuss the unknown secondary component further. We did not find any traces of spectral features belonging to 


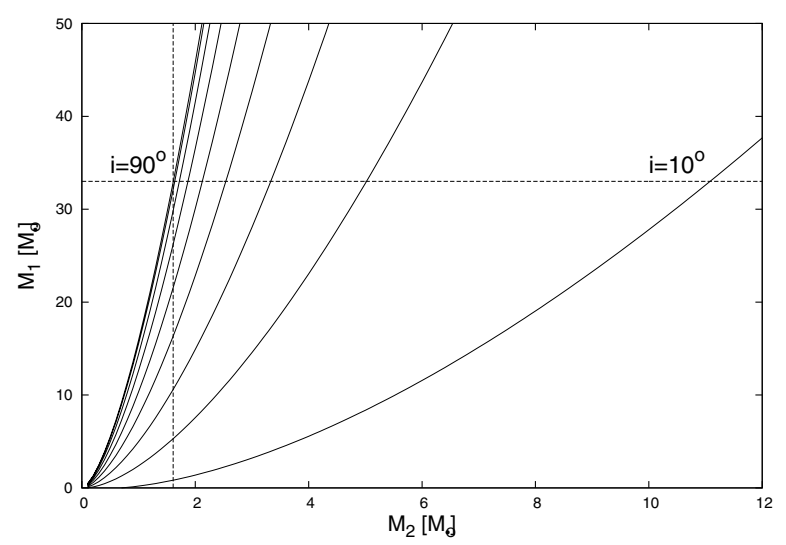

Fig. 5. Mass of the primary as a function of the secondary mass, derived by fixing the mass function to the value of Table 2 . Curves were calculated for each orbital inclination from $90^{\circ}$ to $10^{\circ}$ in steps of $10^{\circ}$. Dotted lines indicate the primary mass, $M_{1}=33 M_{\odot}$, and the minimum possible secondary mass, $M_{2}=1.61 M_{\odot}$, for reference.

the companion. Since we have only determined the mass function $(F(M)$, in Table 2), which relates both masses and the orbital inclination, it is not possible to determine the secondary (minimum) mass unequivocally, but some analysis can be done. In Fig. 5, we depict how the primary mass $\left(M_{1}\right)$ varies as a function of the secondary mass $\left(M_{2}\right)$ and the orbital inclination $(i)$. If we assume that the mass of the primary O8.5 Ib-II star is $33 M_{\odot}$ (average of both calibrations in Martins et al. 2005), it can be inferred that no secondary mass lower than $1.61 M_{\odot}$ is possible. This limit is near the high end of the neutron-star (NS) range. In a recent work, Kiziltan et al. (2013) discuss the NS mass distribution. They find that NS masses peak at $1.33 M_{\odot}$ and $1.55 M_{\odot}$ depending whether they are in NS-NS or NS-white dwarf (WD) binary systems, respectively. Özel et al. (2012) also discuss the NS masses in other populations of binaries, obtaining a mean of $1.28 M_{\odot}$ and a dispersion of $0.24 M_{\odot}$ in eclipsing binaries with high-mass primaries (six values from Rawls et al. 2011). However, there are some NSs with higher values: Vela X-1 $\left(1.84 \pm 0.06 M_{\odot}\right.$, Rawls et al. 2011); PSR J1748-2446I $\left(M=1.91+0.02-0.10 M_{\odot}\right.$, Kiziltan et al. 2013); PSR J1614-2230 $\left(M=1.97 \pm 0.04 M_{\odot}\right.$ Demorest et al. 2010); PSR B1516+02B $\left(M=2.10 \pm 0.19 M_{\odot}\right.$, Kiziltan et al. 2013); and possibly the "Black Widow" pulsar PSR B1957+20 with $M=2.40 \pm 0.12 M_{\odot}$ (van Kerkwijk et al. 2011).

Since the $\mathrm{H} \alpha$ line varies but not in relation to the orbital period, we looked for superorbital modulation as found in other similar binary systems. We found a periodicity of about $285 \mathrm{~d}$ in the EW (considering the absorption and emission components as a whole), but it should be confirmed by further observations. A relationship between superorbital and orbital periods was discussed by Corbet \& Krimm (2013) for a wide variety of X-ray binaries. Our preliminary superorbital period is nearly 30 times the orbital one, which is very far from the relation that seems to exist for the wind-accretion high-mass X-ray binaries, but closer to the one for Roche-lobe overflow powered systems (see Fig. 6).

Acknowledgements. We thank the referee for comments that helped to improve the paper. We thank the directors and staffs of CASLEO, LCO, and ESO La Silla for the use of their facilities and their kind hospitality during the observing runs. CASLEO is operated under agreement between CONICET and the Universities of La Plata, Córdoba and San Juan, Argentina. R.H.B. acknowledges FONDECYT Project No. 1140076. J.I.A. aknowledges financial support from FONDECYT Project No. 11121550. J.M.A., A.S. and E.J.A. acknowledge

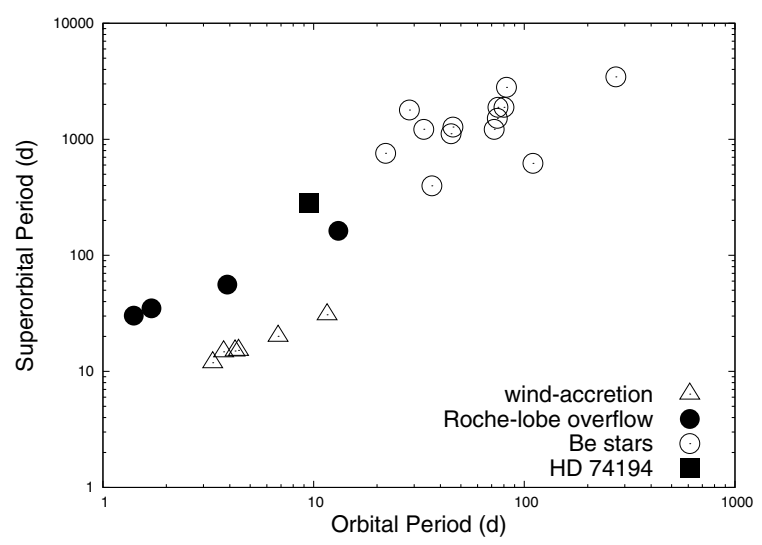

Fig. 6. Position of HD 74194 in the superorbital- vs. orbital-period plot for wind accretion, Roche-lobe overflow systems, and Be stars taken from Corbet \& Krimm (2013) and Rajoelimanana et al. (2011).

support from the Spanish Ministry for Economy and Competitiveness and FEDER funds through grant AYA2013-40611-P. This research made use of NASA's Astrophysics Data System and the SIMBAD database, operated at the CDS, Strasbourg, France.

\section{References}

Akeson, R. L., Chen, X., Ciardi, D., et al. 2013, PASP, 125, 989

Barbá, R., Gamen, R., \& Morrell, N. 2006, ATel, 819, 1

Barbá, R. H., Gamen, R., Arias, J. I., et al. 2010, in Rev. Mex. Astron. Astrofis. Conf. Ser., 38, 30

Barthelmy, S. D., Romano, P., Burrows, D. N., et al. 2009, ATel, 2178, 1

Bertiau, F. C., \& Grobben, J. 1969, Ricerche Astronomiche, 8, 1

Bozzo, E., Romano, P., Ducci, L., Bernardini, F., \& Falanga, M. 2015, Adv. Space Res., 55, 1255

Conti, P. S., Leep, E. M., \& Lorre, J. J. 1977, ApJ, 214, 759

Corbet, R. H. D., \& Krimm, H. A. 2013, ApJ, 778, 45

Demorest, P. B., Pennucci, T., Ransom, S. M., Roberts, M. S. E., \& Hessels, J. W. T. 2010, Nature, 467, 1081

Feast, M. W., Thackeray, A. D., \& Wesselink, A. J. 1955, MmRAS, 67, 51

Gies, D. R. 1987, ApJS, 64, 545

Gotz, D., Schanne, S., Rodriguez, J., et al. 2006, ATel, 813, 1

Götz, D., Falanga, M., Senziani, F., et al. 2007, ApJ, 655, L101

Hadrava, P. 2004, Publications of the Astronomical Institute of the Czechoslovak Academy of Sciences, 92, 1

Kalogera, V. 1996, ApJ, 471, 352

Karino, S. 2010, A\&A, 523, A90

Kiziltan, B., Kottas, A., De Yoreo, M., \& Thorsett, S. E. 2013, ApJ, 778, 66

Maíz Apellániz, J., Walborn, N. R., Galué, H. Á., \& Wei, L. H. 2004, ApJS, 151, 103

Mangano, V., Romano, P., Barthelmy, S. D., et al. 2011, ATel, 3586, 1

Marraco, H. G., \& Muzzio, J. C. 1980, PASP, 92, 700

Martins, F., Schaerer, D., \& Hillier, D. J. 2005, A\&A, 436, 1049

Masetti, N., Bassani, L., Bazzano, A., et al. 2006, ATel, 815, 1

McSwain, M. V., Boyajian, T. S., Grundstrom, E. D., \& Gies, D. R. 2007, ApJ, 655,473

Mereghetti, S., Sidoli, L., Paizis, A., \& Gotz, D. 2006, ATel, 814, 1

Özel, F., Psaltis, D., Narayan, R., \& Santos Villarreal, A. 2012, ApJ, 757, 55

Rajoelimanana, A. F., Charles, P. A., \& Udalski, A. 2011, MNRAS, 413, 1600

Rawls, M. L., Orosz, J. A., McClintock, J. E., et al. 2011, ApJ, 730, 25

Romano, P. 2015, J. High Energy Astrophys., 7, 126

Romano, P., Sidoli, L., Cusumano, G., et al. 2009, MNRAS, 392, 45

Romano, P., Barthelmy, S. D., Baumgartner, W. H., et al. 2010, ATel, 2520, 1

Romano, P., Lien, A. Y., Evans, P. A., et al. 2013, ATel, 5190, 1

Romano, P., Krimm, H. A., Palmer, D. M., et al. 2014, A\&A, 562, A2

Scargle, J. D. 1982, ApJ, 263, 835

Sidoli, L. 2013, ArXiv e-prints [arXiv: 1301.7574]

Sidoli, L., Romano, P., Ducci, L., et al. 2009, MNRAS, 397, 1528

Sota, A., Maíz Apellániz, J., Morrell, N. I., et al. 2014, ApJS, 211, 10

van der Hucht, K. A. 2001, New Astron. Rev., 45, 135

van Kerkwijk, M. H., Breton, R. P., \& Kulkarni, S. R. 2011, ApJ, 728, 95

Walborn, N. R. 1973, AJ, 78, 1067

Page 5 is available in the electronic edition of the journal at http://www . aanda.org 
R. Gamen et al.: The eccentric short-period orbit of the supergiant fast X-ray transient HD 74194 (=LM Vel)

Table 3. Outbursts of HD 74194 found in the literature.

\begin{tabular}{clcccc}
\hline \hline Id. & Date & Cite & Mission & HJD & $\phi$ \\
\hline 1 & $2003-07-01$ & Götz et al. (2007) & INTEGRAL & 2452822.333 & 0.07 \\
2 & $2006-05-15$ & Götz et al. (2007) & INTEGRAL & 2453871.271 & 0.98 \\
3 & $2006-10-04$ & Götz et al. (2007) & Swift & 2454013.115 & 0.84 \\
4 & $2008-07-05$ & Romano et al. (2009) & Swift & 2454653.385 & 0.93 \\
5 & $2008-09-21$ & Sidoli et al. (2009) & Swift & 2454730.828 & 0.05 \\
6 & $2009-08-28$ & Barthelmy et al. (2009) & Swift & 2455072.453 & 0.84 \\
7 & $2010-03-28$ & Romano et al. (2010) & Swift & 2455284.162 & 0.03 \\
8 & $2011-08-25$ & Mangano et al. (2011) & Swift & 2455798.537 & 0.92 \\
9 & $2013-07-02$ & Romano et al. (2013) & Swift & 2456475.841 & 0.89 \\
\hline
\end{tabular}

